\section{A FORTRAN IV function for computing critical values of ordered half-normal scores}

\section{WILLIAM P. DUNLAP and N. CLAYTON SILVER Tulane University, New Orleans, Louisiana}

If the standard normal distribution $(M=0.0, S D=$ 1.0 ) is modified by transforming all negative values (those below the mean) to positive values, the half-normal distribution results. Dunlap and Myers (1984) described a FORTRAN program that computes the expected values of ordered half-normal scores, which are useful for halfnormal probability plots. Values that may prove to be even more useful are the critical values of ordered half-normal distribution, which can be used directly in tests of significance. These critical values have specific applications for hypothesis testing, two of which are discussed here.

When many correlations in a matrix are tested for significance, repeated use of standard tests such as those based on $F$ or on Fisher's $z$ transformation involves repeated risks of a Type I error; therefore, some correlations may appear significant by chance alone. Hills (1969) and Stavig and Acock (1976) proposed tests of significance for correlations in a matrix that attempt to control overall Type I error rates, unlike repeated $F$ or $z$ tests. The rank adjusted method (Stavig \& Acock, 1976) orders correlations in terms of absolute value, transforms these correlations via Fisher's $z$ transformation, and then calculates the corresponding normal deviates by dividing by the standard error of Fisher's $z, 1 /(N-3)^{1 / 2}$. Because the process started from the absolute values of the original correlations, the resulting scores should have had the ordered half-normal distribution. Therefore, Stavig and Acock (1976) recommended subtracting the corresponding expected ordered half-normal score and comparing the difference to the critical value of the standard normal distribution. The problem is that comparing these adjusted $z$ scores to the standard normal distribution will result in a conservative test (Silver, 1988).

If instead, each rank ordered $z$ score is compared to the corresponding critical value of the half-normal distribution, a much improved test results. If the $i$ th $z$ score equals or exceeds the $i$ th critical value of the half-normal distribution, it is considered significant. This procedure not only has been shown to control Type I errors well, but exhibits greater power than either the multistage Bonferroni (Larzelere \& Mulaik, 1977) or Stavig and Acock (1976) procedures (Silver, 1988).

A second example of the use of ordered half-normal critical values applies to the analysis of a $2^{5}$ factorial design, where each of five experimental variables has two levels. For such a design, there are 31 tests of significance

Correspondence may be addressed to William P. Dunlap, Department of Psychology, Tulane University, New Orleans, LA 70118. for the various main effects and interactions. With so many tests of significance, some effects are likely to be quite large by chance alone, and several might be significant if tested in the customary way, by analysis of variance. Because all effects in a $2^{5}$ are linear contrasts of the cell means, and each is based on one degree of freedom, Daniel (1959) and Zahn (1975a, 1975b) have proposed judging significance by plotting the ordered absolute values of the standardized contrasts on half-normal probability paper, where departures from a straight line would indicate an effect larger than that expected by chance alone. Such an approach may be particularly useful in experiments with only one replication, where there is no direct estimate of error. Making such judgments with ordered half-normal critical values, rather than visual inspection of plots, should vastly improve these procedures. Other potential applications for these critical values are mentioned by Dunlap and Myers (1984).

\section{The Program}

The half-normal probability function was calculated from the normal probability function, using the algorithm of Divgi (1979). Simpson's rule was used to numerically integrate the function as described by Dunlap and Myers (1984). The program continues to integrate the ordered half-normal distribution until the area exceeds one minus alpha; thus, the critical value is exceeded. Then a process of adjustment ensues, in which the correct critical value is estimated by linear interpolation, and small areas are either added or subtracted from the total area to refine the estimate. This adjustment procedure continues until the integral up to the critical value agrees with one minus alpha to five decimal places (for a fairly complete tabulation of critical values generated please see Appendix A).

\section{Accuracy}

To confirm the accuracy of the resulting critical values, a Monte Carlo simulation was performed. Empirical alpha levels for each critical value were obtained by counting the number of times 10,000 randomly generated ordered half-normal scores surpassed these critical values. Empirical alpha values approximated theoretical alpha values well (range $= \pm .0004$ ) for critical values of small to moderate numbers of ordered half-normal scores. There was, however, a slight conservative trend for larger numbers of ordered half-normal scores (e.g., $N=100$ ). The present program is probably accurate to five decimal places, because it uses the same computational scheme as that of Dunlap and Brown (1983) and Dunlap and Myers (1984). The FORTRAN program is given in Appendix B.

\section{Program Availability}

These functions are written in standard FORTRAN IV and run on an IBM $3081 \mathrm{GX}$ computer. On this system 
the Unit 9 is assigned by default to the keyboard and CRT. Listings of these functions can be obtained at no cost from William P. Dunlap, Department of Psychology, Tulane University, New Orleans, LA 70118.

\section{REFERENCES}

DANIEL, C. (1959). Use of half-normal plots in interpreting factorial two-level experiments. Technometrics, 1, 311-341.

DivgI, D. R. (1979). Calculation of univariate and bivariate normal probability functions. Annals of Statistics, 7, 903-910.

DunlaP, W. P., Brown, S. G. (1983). FORTRAN IV functions to compute expected normal scores. Behavior Research Methods \& Instrumentation, 15, 395-397.

DUNLAP, W. P., MYERS, L. (1984). FORTRAN IV functions for com- puting expected half-normal scores. Behavior Research Methods, Instruments, \& Computers, 16, 401-404.

HILLs, M. (1969). On looking at large correlation matrices. Biometrika, 56, 249-253.

Larzelere, R. E., \& Mulaik, S. A. (1977). Single-sample tests for many correlations. Psychological Bulletin, 84, 557-569.

Silver, N. C. (1988). Type I errors and power of tests of correlations in a matrix. Unpublished doctoral dissertation, Tulane University, New Orleans, LA.

Stavig, G. R., \& Acock, A. C. (1976). Evaluating the degree of dependence for a set of correlations. Psychological Bulletin, 83, 236241.

ZAHN, D. A. (1975a). An empirical study of the half-normal plot. Technometrics, 17, 201-211.

ZAHN, D. A. (1975b). Modifications of and revised critical values for the half-normal plot. Technometrics, 17, 189-200. 


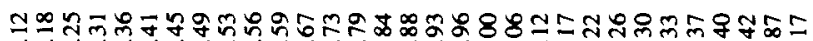

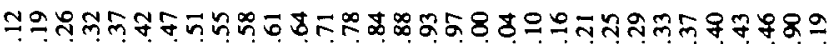

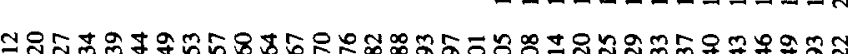

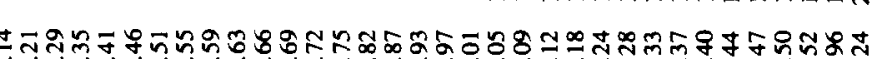

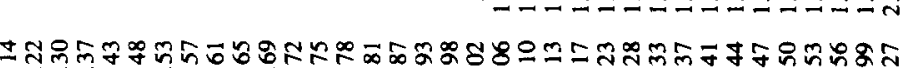
सกตก

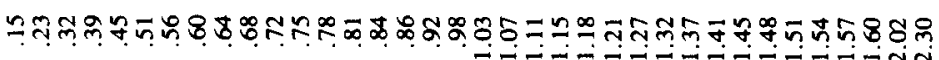

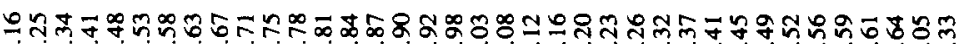

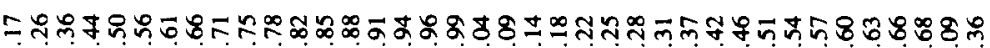

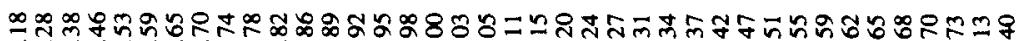

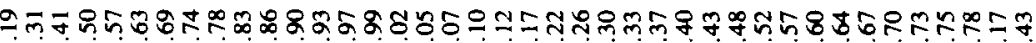

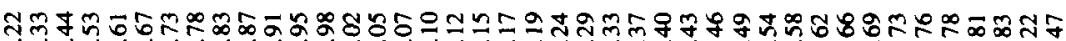

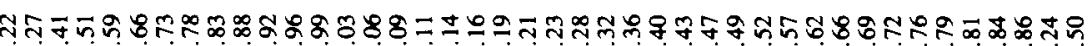
กุจุ

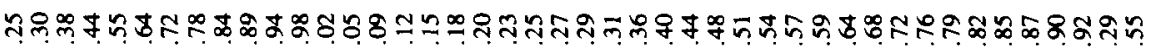

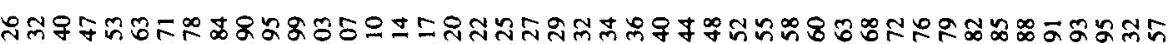

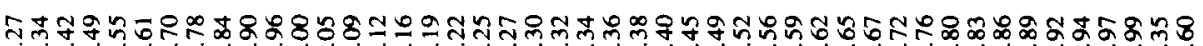

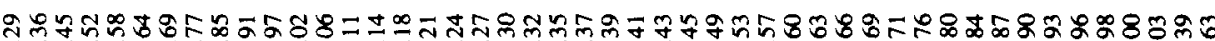

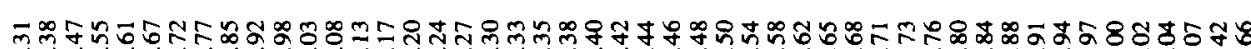

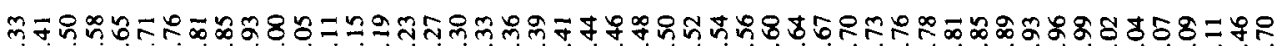

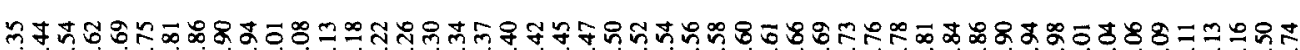

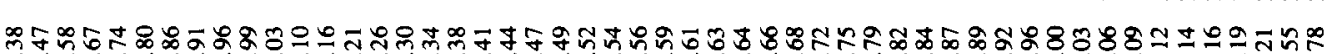

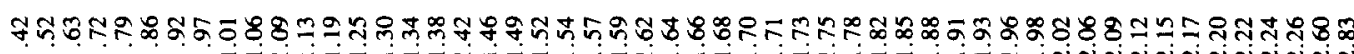

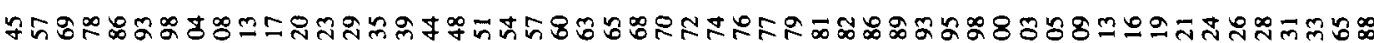
7. -

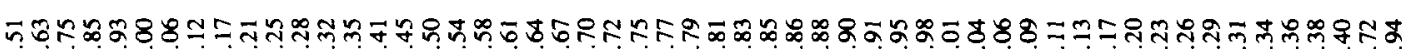

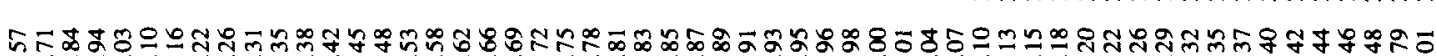

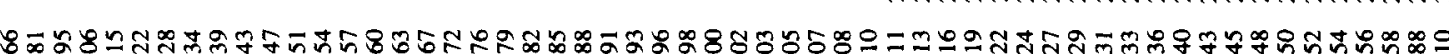
Ji

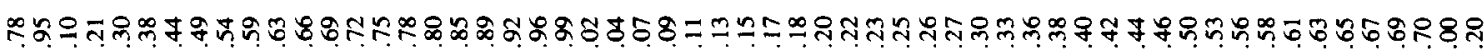

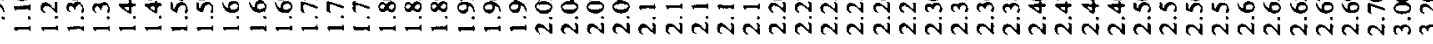

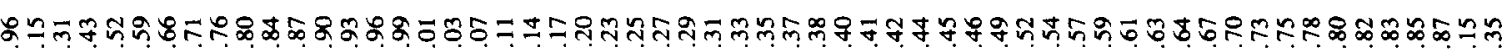
- .

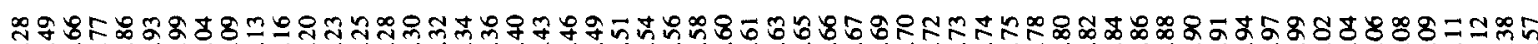

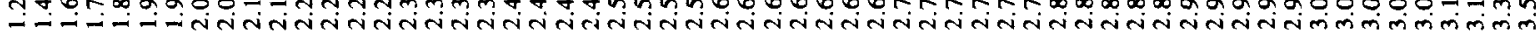

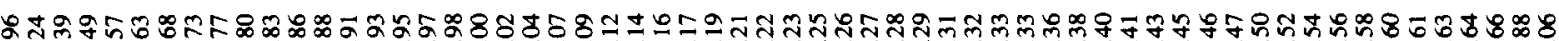

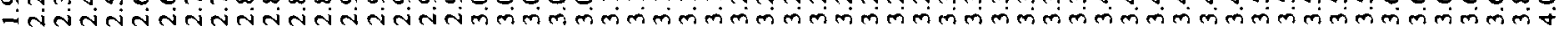




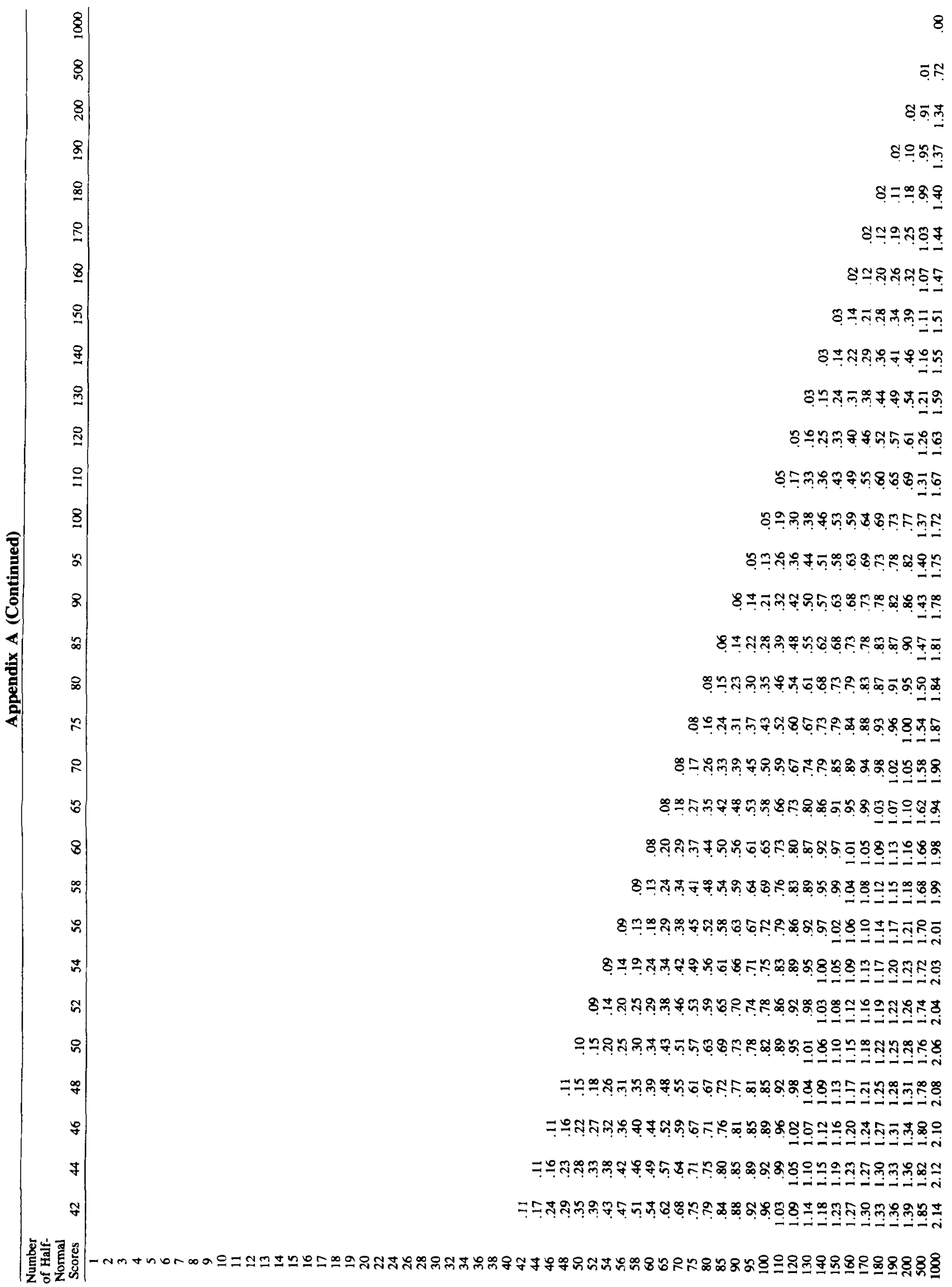


C MAIN ROUTINE FOR NUMERICAL CALCULATION OF CRITICAL VALUES

C OF ORDERED HALF NORMAL SCORES DOUBLE PRECISION HNSCR WRITE $(9,2)$ FORMAT ('ENTER N, ALPHA (ENTER 0,0 TO QUIT)') $\operatorname{READ}\left(9,{ }^{\star}\right) \mathrm{N}, \mathrm{A}$ IF (N.EQ.O) STOP

DO $4 \mathrm{~J}=1, \mathrm{~N}$ $\mathrm{CV}=\operatorname{HNSCR}(\mathrm{J}, \mathrm{N}, \mathrm{A})$ WRITE $(9,3) \mathrm{N}, \mathrm{J}, \mathrm{CV}, \mathrm{A}$

3 FORMAT (' $\mathrm{CV}\left({ }^{\prime}, 2 \mathrm{I} 5, '\right)=\prime$, F12.5,' ALPHA $=\prime$, F8.4)

4 CONTINUE

GO TO 1

END

C

FUNCTION $\operatorname{HNSCR}(\mathrm{J}, \mathrm{N}, \mathrm{A})$

IMPLICIT DOUBLE PRECISION $(A-\mathrm{H}, \mathrm{O}-\mathrm{Z})$

C COMPUTES THE CRITICAL VALUE OF THE JTH OF $N$

C ORDERED HALF NORMAL SCORES

C COMPUTE THE CONSTANT OF INTEGRAL IN LOG FORM $\mathrm{CA}=1 .-\mathrm{A}$ $\mathrm{C}=\mathrm{FACL}(\mathrm{N})-\mathrm{FACL}(\mathrm{J}-1)-\mathrm{EACL}(\mathrm{N}-\mathrm{J})$ HNSCR $=0$. $S T R=0$. $F I N=0$.

C FIND LOWER (STR) AND UPPER (FIN) LIMITS OF INTEGRATION DO $1 \mathrm{I}=1,50$

$X I=I$

$X I=X I \star .14$

$E U N C=F U N(X I, C, J, N)$

IF (STR.EQ.0. . AND.FUNC.GT.1.E-7) STR=XI

1 CONTINUE

$2 \quad$ FIN $=X I$

$S T R=S T R-.14$

IF (STR.GE.0.) GOTO 3

$\mathrm{STR}=0$.

$\mathrm{FIN}=.28$

CONT INUE

$\mathrm{W}=(\mathrm{EIN}-\mathrm{STR}) / 100$.

$X L=S T R$

$F L=F U N(X L, C, J, N)$

C BEGIN SIMPSON'S RULE INTEGRATION

DO $4 \quad I=1,101$

$\mathrm{XU}=\mathrm{XL}+\mathrm{W}$

$X M=(X U+X L) / 2$.

$E M=F U N(X M, C, J, N)$

$\mathrm{FU}=\mathrm{FUN}(\mathrm{XU}, \mathrm{C}, \mathrm{J}, \mathrm{N})$

$X L S T=X L$

$\mathrm{HLST}=\mathrm{HNSCR}$

$\mathrm{HNSCR}=\mathrm{HNSCR}+\left(\mathrm{FU}+4 \cdot{ }^{\star} \mathrm{FM}+\mathrm{FL}\right){ }^{\star} \mathrm{W} / 6$.

IF (HNSCR.GT.CA) GOTO 5

$\mathrm{XL}=\mathrm{XU}$

$F L=F U$

4 CONVERGE ON CRITICAL VALUE

C ESTIMATE CV

$5 \quad \mathrm{CV}=\mathrm{XLST}+(\mathrm{XU}-\mathrm{XLST}) *(\mathrm{CA}-\mathrm{HLST}) /(\mathrm{HNSCR}-\mathrm{HLST})$

$\mathrm{FCV}=\mathrm{FUN}(\mathrm{CV}, \mathrm{C}, \mathrm{J}, \mathrm{N})$

$X M=(C V+X U) / 2$.

$\mathrm{W}=\mathrm{ABS}(\mathrm{XM}-\mathrm{CV})$

$F M=F U N(X M, C, J, N)$

$A R E A=W / 3 .{ }^{\star}(E C V+4.0 \star F M+E U)$

IF (HNSCR. GT . CA) AREA $=-($ AREA)

ANEW $=$ HNSCR+AREA

IE (ABS (ANEW-CA) . LE. 0.000001D0) GOTO 7

IF (ABS (CV-XLST) . GT . ABS (XU-CV)) GOTO 6

$\mathrm{XU}=\mathrm{CV}$

HNSCR $=$ ANEW

$\mathrm{FU}=\mathrm{FCV}$

GOTO 5

GOTO 5 


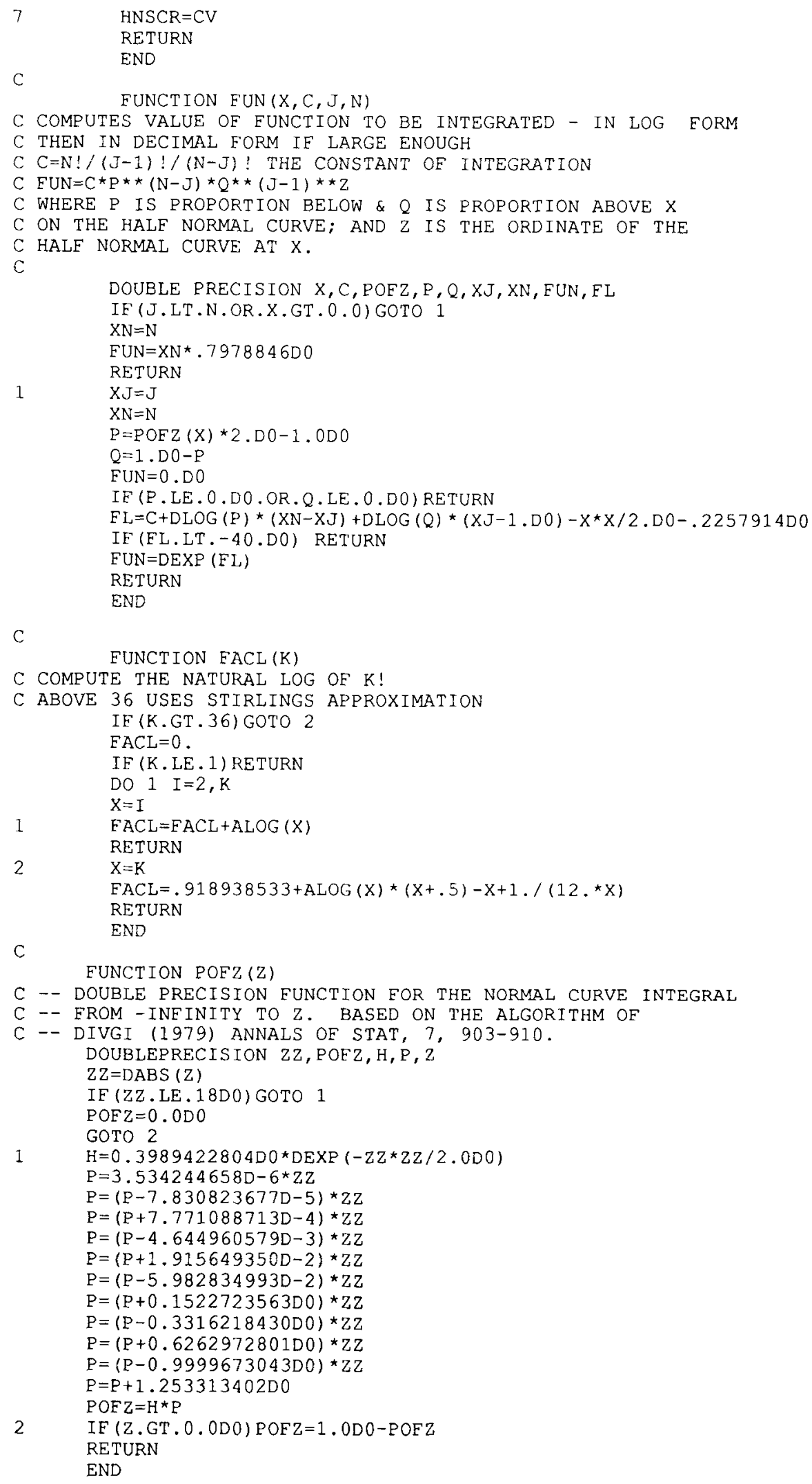

